\title{
Environmental Sampling for Detection of Mycobacterium avium ssp. paratuberculosis on Large California Dairies
}

\author{
R. D. Berghaus, ${ }^{\star 1}$ T. B. Farver, ${ }^{*}$ R. J. Anderson,† C. C. Jaravata, ${ }^{\star}$ and I. A. Gardnerł \\ ${ }^{*}$ Department of Population Health and Reproduction, School of Veterinary Medicine, University of California, Davis 95616 \\ †California Department of Food and Agriculture, Animal Health Branch, Sacramento 95814 \\ ‡Department of Medicine and Epidemiology, School of Veterinary Medicine, University of California, Davis 95616
}

\begin{abstract}
Environmental samples collected from each of 3 locations on 23 large California dairies were cultured to evaluate the utility of this approach for identifying herds infected with Mycobacterium avium ssp. paratuberculosis. Results were compared with concurrent ELISA testing of $\geq 60$ animals in each herd, and with previously performed individual and pooled fecal cultures of 60 animals. The estimated proportions of infected herds did not differ significantly among the testing methods (environmental sampling, $74 \%$; previous fecal culture, 70\%; and concurrent ELISA testing, 65\%). Measures of agreement between environmental sampling and the results of previous fecal cultures were 70\% (observed agreement), 85\% (positive agreement), $62 \%$ (negative agreement), and 0.47 (kappa), whereas agreement between environmental sampling and concurrent ELISA testing was 65,75 , and $43 \%$, and 0.19 , for the same measures, respectively. The proportion of positive environmental samples on each farm was significantly correlated with the proportion of seropositive animals $(r=0.53)$, suggesting that environmental sampling may also provide a qualitative estimate of within-herd prevalence. Of the sampling locations that were evaluated, samples of lagoon water (15/23; 65\%) were significantly more likely to yield a positive result than were composite manure samples $(8 / 22 ; 36 \%)$ collected from the sick/fresh cow pen or from the alleyway (9/23; 39\%) where cows exited from the milking parlor. Environmental sampling was an effective and inexpensive method of identifying herds infected with Mycobacterium avium ssp. paratuberculosis.
\end{abstract}

Key words: Mycobacterium paratuberculosis, Johne's disease, environmental sampling, dairy

\section{INTRODUCTION}

Correct classification of the infection status of cattle herds with Mycobacterium avium ssp. paratuberculosis

Received August 31, 2005.

Accepted October 31, 2005.

${ }^{1}$ Corresponding author: rdberghaus@ucdavis.edu
(MAP) and estimation of within-herd prevalence in infected herds are important components of control programs, herd status programs, and health surveys for Johne's disease. Several herd-testing strategies based on single and multiple tests, including milk and serum ELISA (Wells et al., 2002; Hendrick et al., 2005), and individual and pooled fecal culture (van Schaik et al., 2003; Tavornpanich et al., 2004) have been considered in different studies. Although the goal of testing is to obtain high herd sensitivity and specificity at reasonable cost, errors in classification of MAP status occur because many herds have low prevalence $(<5 \%)$, a finite sample of the herd is tested, and diagnostic tests have low to moderate sensitivity in subclinically infected animals and (with the likely exception of culture) are imperfectly specific.

Recently, the role of environmental sampling for herd classification has been evaluated in several studies (Raizman et al., 2004; Fyock et al., 2005; USDA, 2005a). Potential advantages to this approach include lower cost than ELISA testing or individual fecal culture, ease of sampling, no additional handling of individual animals, and specificity that is near $100 \%$. If testing of multiple environmental samples per herd allowed for a qualitative estimate of within-herd prevalence, then the utility of the approach would be increased.

In a study of Minnesota dairy operations, Raizman et al. (2004) found that culture of environmental samples resulted in herd-status classifications similar to those obtained when fecal cultures were pooled from up to 100 animals in each herd, with cow alleyways and manure storage areas yielding the greatest proportion of positive results. Sampling methods in that study varied among farms and cultures were performed on several different substrates, including manure, bedding, and soil. In the National Animal Health Monitoring System's Dairy 2002 study, environmental sampling was focused on collecting 5 composite manure samples from areas on each operation where a majority of cows had the opportunity to contribute to the sample. Many different veterinary medical officers performed the sampling and the areas that were sampled were inconsistent across farms. 
The US Animal Health Association's Voluntary Johne's Disease Herd Status Program for cattle (Bulaga, 1998) and the USDA's Voluntary Bovine Johne's Disease Control Program (USDA, 2005b) were both developed to provide guidelines for state-administered paratuberculosis programs. Entry into the herd certification component of these programs currently requires ELISA testing of 30 animals in their second lactation or later, with follow-up fecal cultures for any seropositive animals. If producers wish to advance to higher certification levels, they must test a sufficient number of animals using ELISA and fecal culture to have a 95\% probability of detecting one or more infected cows in a herd with $2 \%$ prevalence. As this requires a substantial increase in the number of animals tested (all animals in their second lactation or later for herds with $\leq 500$ cows), it is to the producer's benefit to detect MAP during the initial round of testing if it is present in the herd.

Consequently, improving the sensitivity of entrylevel testing without substantially increasing the cost could improve the utility of these programs without discouraging producers from participation. With this goal in mind, environmental sampling has recently been approved as a method of entry-level testing in the USDA's Voluntary Bovine Johne's Disease Control Program (USDA, 2005b).

The objectives were to 1 ) evaluate a standardized environmental manure sampling protocol by a single veterinary medical officer as a method of identifying the MAP infection status of several large California dairies, and 2) compare the cost and effectiveness of environmental sampling with concurrent ELISA testing results, and with the results of previous fecal culture testing of individual animals that had been performed on the same farms.

\section{MATERIALS AND METHODS}

\section{Selection of Herds}

Twenty-three free-stall dairies with a median herd size of 680 cows (range: 350 to 2,500 cows) were asked to participate in the study to evaluate the use of environmental sampling for detection of MAP. These operations were all clients of a single large veterinary practice in California's Central Valley, and were a subset of herds that had participated in a previous study of pooled culture methods for the detection of MAP (Tavornpanich et al., 2004). Consequently, an advantage of enrolling these herds was the availability of well-documented recent historical information with respect to their MAP infection status.

\section{Environmental Sample Collection}

Environmental sampling was conducted by a veterinary medical officer (R. J. Anderson) between January 23, 2004, and June 11, 2004. Composite manure or effluent samples were collected from farms in each of 3 locations: the return alley where cows exit from the milking parlor, the alleyways of the sick/fresh cow pen, and the wastewater storage lagoon. The return alley was sampled immediately after milking by taking 10 $\mathrm{mL}$ grab samples of manure at approximately 1-m intervals while walking along its length. Grab samples were combined in a 2 -L plastic bucket and mixed thoroughly with a wooden tongue depressor before transferring $40 \mathrm{~mL}$ of the slurry to a sterile $50-\mathrm{mL}$ polypropylene conical tube. If the alley had recently been flushed with water, then areas where manure had not been completely removed were sampled. The goal during alleyway sampling was to collect manure that was perceived as a homogeneous mixture; discernible individual fecal pats were deliberately avoided.

Manure was collected from the sick/fresh cow pen by sampling from crossover alleys and the areas around water tanks where cattle congregate and flushing tends to be incomplete. These locations were typically rectangular, and ranged in area from approximately 15 to 30 $\mathrm{m}^{2}$. Within these areas, one $10-\mathrm{mL}$ grab sample was collected for every 1 to $2 \mathrm{~m}^{2}$ of surface area, and the samples from all such areas in the pen were combined in a 2-L plastic bucket. In addition, manure within 0.5 $\mathrm{m}$ of the outer structural edges of sampling areas was typically avoided because it had a tendency to appear drier and less "fresh" than that in the middle of the sampling areas where cow traffic was more consistent. The combined slurry samples were mixed as described for the return alleyway. Plastic mixing buckets were washed between sampling locations, sanitized by brushing with an o-phenylphenol disinfectant, and thoroughly rinsed with fresh water. A new latex glove was used to collect the grab samples for each different composite sampling location and a new tongue depressor was used for mixing.

Lagoons were sampled at a location along their perimeter where it was possible to safely approach the water's edge. Floating debris and solid materials were swept aside where necessary, and a single sample of wastewater was collected by immersing an autoclaved 500-mL, wide-mouth polypropylene container up to 10 $\mathrm{cm}$ beneath the surface. All containers were capped and refrigerated until the samples were processed.

\section{Laboratory Testing}

Culture of Environmental Samples. Environmental samples were processed by the Dairy Food Safety 
Laboratory at the University of California-Davis within $48 \mathrm{~h}$ of sample collection. Manure samples collected from the return alleyways and sick/fresh cow pens were cultured using an adaptation of the method that has been recommended by the USDA National Veterinary Services Laboratory (Stabel, 1997). Briefly, $2 \mathrm{~g}$ of the fecal slurry was added to $35 \mathrm{~mL}$ of sterile water and mixed thoroughly before allowing the tube to stand at room temperature for $30 \mathrm{~min}$. The supernatant was removed and centrifuged at $1,700 \times g$ for $20 \mathrm{~min}$, after which the pellet was resuspended in $30 \mathrm{~mL}$ of brain heart infusion broth containing $0.9 \%$ hexadecylpyridinium chloride. The solution was incubated overnight at $37^{\circ} \mathrm{C}$, and then centrifuged at $1700 \times g$ for 20 min, after which the pellet was resuspended in $1 \mathrm{~mL}$ of an antibiotic wash solution containing $100 \mu \mathrm{g} / \mathrm{mL}$ of nalidixic acid, $100 \mu \mathrm{g} / \mathrm{mL}$ of vancomycin, and $50 \mu \mathrm{g} / \mathrm{mL}$ of amphotericin B. After an overnight incubation at $37^{\circ} \mathrm{C}, 4$ tubes of Herrold's egg yolk medium containing mycobactin $\mathrm{J}$, nalidixic acid $(50 \mu \mathrm{g} / \mathrm{mL})$, vancomycin $(50 \mu \mathrm{g} / \mathrm{mL})$, and amphotericin B $(50 \mu \mathrm{g} / \mathrm{mL})$ were inoculated with $200 \mu \mathrm{L}$ of the suspension, as was one tube without mycobactin J. Tubes were incubated at $37^{\circ} \mathrm{C}$ horizontally for $1 \mathrm{wk}$ and then incubated vertically with tightened caps for up to 12 wk. Lagoon samples were treated similarly, except that $2 \mathrm{~mL}$ of the lagoon water was processed instead of $2 \mathrm{~g}$ of feces. Colonies were identified based on their morphology, acid-fast staining characteristics, and mycobactin dependency, with additional confirmation based on amplification of a section of the IS900 gene sequence by PCR (Vary et al., 1990).

PCR Confirmation of Positive Environmental Cultures. The Dairy Food Safety Laboratory used realtime PCR with fluorescence resonance energy transfer probes (Roche Lightcycler, Roche Diagnostics Corp., Indianapolis, IN) to amplify a segment of the IS900 insertion sequence of MAP. Briefly, suspect colonies were collected from slants using a sterile pick and placed in $5 \mathrm{~mL}$ of Middlebrook broth with mycobactin $\mathrm{J}$ for $1 \mathrm{wk}$ at $37^{\circ} \mathrm{C}$. Then, DNA was extracted from $65 \mu \mathrm{L}$ of the broth that was placed onto a commercially available card medium (Whatman FTA card, Whatman, Clifton, NJ). The following mix of reagents was used in the PCR reaction: Roche Lightcycler Fast Start DNA Master Hybridization probes kit, $3 \mathrm{mM} \mathrm{MgCl}_{2}, 0.25 \mathrm{mg} / \mathrm{mL}$ of BSA, $0.3 \mu M$ Red 640 probe (5'- GGATCGCTGTGTAAGGACACG-3'), $0.2 \mu M$ fluorescein (5'-ACCGCTAATTGAGAGATGCGA-3'), $0.5 \mu M$ forward primer (5'-CTTGAAGGGTGTTCGG-3'), and $0.5 \mu M$ reverse primer (5'-ACCGTGTTGGACAGAC-3'), $1 \mu \mathrm{L}$ of uracilDNA-glycosylase, and $1 \times$ Lightcycler Fast Start DNA master hybridization probes mix. All PCR reagents were from Roche Diagnostics Corp. Polymerase chain reaction conditions were: $95^{\circ} \mathrm{C}$ for 10 min followed by
50 cycles at $95^{\circ} \mathrm{C}$ for $<1 \mathrm{~s}, 58^{\circ} \mathrm{C}$ for $10 \mathrm{~s}$, and $72^{\circ} \mathrm{C}$ for $9 \mathrm{~s}$. After amplification, a melting cycle was performed with denaturation at $95^{\circ} \mathrm{C}$ for $30 \mathrm{~s}$, a decrease to $38^{\circ} \mathrm{C}$ for $30 \mathrm{~s}$, and a slow rise $\left(0.2^{\circ} \mathrm{C} / \mathrm{s}\right)$ to $80^{\circ} \mathrm{C}$ for $<1 \mathrm{~s}$ with continuous monitoring of fluorescence. A positive result was based on the presence of a melting curve temperature between 62 to $63^{\circ} \mathrm{C}$, corresponding to hybridization between the probes and the IS900 sequence.

Positive and negative controls were included with each run. The MAP controls were strain ATCC 43544 or ATCC 43545, and negative controls consisted of the PCR mix spiked with Middlebrook broth.

ELISA Testing. Serum was collected by a veterinary medical officer from a systematic random sample of $\geq 60$ lactating cows in each herd and a commercially available Johne's ELISA test (HerdCheck, Idexx Laboratories Inc., Westbrook, ME) was performed by the California Animal Health and Food Safety Laboratory at the University of California-Davis. Serum samples were collected on a single visit during the week of environmental sample collection for 21 of the herds, whereas samples in the remaining 2 herds (herds 212 and 230) were collected from cows that were routinely tested at dry-off between March 1 and April 30, 2004. These latter 2 herds were also participating in a Johne's demonstration herd project, and ELISA testing that was already being performed for that study was utilized. Although at least 60 samples were collected in each herd, broken tubes or hemolytic samples reduced the number of ELISA results that were available in 2 of the herds to 57 and 59 tests, respectively. The ELISA results were measured as the sample optical density (OD) and were recorded as a sample-to-positive (S/P) ratio, where $\mathrm{S} / \mathrm{P}=(\mathrm{OD}$ of unknown sample $-\mathrm{OD}$ of negative-control sample)/(OD of positive-control sample - OD of negative-control sample). Samples were initially assayed in a single well on the ELISA plate. Samples with an S/P ratio $<0.2$ were considered negative, whereas samples with an $\mathrm{S} / \mathrm{P}$ ratio $\geq 0.2$ were reassayed and the mean of the 2 readings was used as the final result. A mean $\mathrm{S} / \mathrm{P}$ ratio $\geq 0.25$ was considered a positive result as recommended by the kit's manufacturer.

\section{Previous Fecal Cultures of Individual Animals.} Fecal culture testing performed in these herds during an earlier study has been described (Tavornpanich et al., 2004). Briefly, fecal samples were collected from a random sample of 60 lactating animals in each herd during the fall of 2001. Fecal samples were cultured both individually and in pools ( 6 pools of 10 individual samples per pool) using Herrold's egg yolk media and an automated liquid culture system (ESP paraJEM culture system II, Trek Diagnostic Systems Inc., Sun Prairie, WI). Decontamination and sample preparation pro- 
Table 1. Data layout for summarizing the agreement between paratuberculosis testing methods on 23 California dairies ${ }^{1}$

\begin{tabular}{llll} 
& \multicolumn{2}{c}{ Test 1} & \\
\cline { 2 - 3 } Test 2 & Positive & Negative & Totals \\
\hline Positive & $a$ & $b$ & $g_{1}$ \\
Negative & $c$ & $d$ & $g_{2}$ \\
Totals & $f_{1}$ & $f_{2}$ & $n$ \\
\hline
\end{tabular}

${ }^{1} a$ represents the number of herds that were identified as positive by both tests, and $d$ represents the number with negative results on both tests. Herds represented by $b$ and $c$ are those with discordant testing results, marginal totals are represented by $f$ and $g$, and $n$ is the total number of herds that were tested.

cedures were based on the method recommended by the National Veterinary Services Laboratory (Stabel, 1997). Herds with at least one positive result on either the individual or pooled fecal cultures were considered to be MAP infected.

\section{Statistical Methods}

Cochran's Q was used to compare the proportions of operations that were identified as positive by the different testing methods, and to compare the proportions of positive samples from each of the 3 environmental sampling locations (Fleiss et al., 2003). Spearman's correlation coefficient was used to evaluate the relationship between the proportion of positive environmental samples on operations and the proportion of seropositive animals.

In evaluating the agreement between 2 testing methods, data were summarized in a $2 \times 2$ arrangement as shown in Table 1, where $a$ represents the number of herds that were identified as positive by both tests, and $d$ represents the number with negative results on both tests. Herds represented by $b$ and $c$ are those with discordant testing results, marginal totals are represented by $f$ and $g$, and $n$ is the total number of herds that were tested. With reference to this table, the proportion of observed agreement $\left(p_{0}\right)$ is a measure of overall concordance, and is defined as $(a+d) / n$. Kappa is a measure of the proportion of agreement beyond that which would be expected due to chance, and is defined as $\left(p_{\mathrm{o}}-p_{\mathrm{e}}\right) /(1$ $\left.-p_{\mathrm{e}}\right)$, where $p_{\mathrm{e}}$ is the proportion of expected agreement, which in turn is defined as $\left(f_{1} g_{1}+f_{2} g_{2}\right) / n^{2}$ (Cohen, 1960).

Because kappa is influenced by prevalence and marginal asymmetry, additional outcome-specific measures of agreement have been recommended to improve the evaluation of agreement for positive and negative outcomes (Cicchetti and Feinstein, 1990). Two such measures include the proportion of positive agreement, which is defined as the number of herds that were identified as positive by both methods divided by the average number identified as positive by either method $[2 a /$ $\left.\left(f_{1}+g_{1}\right)\right]$; and the proportion of negative agreement, which is defined as the number of herds that were identified as negative by both methods divided by the average number that were identified as negative by either method $\left[2 d /\left(f_{2}+g_{2}\right)\right]$. Exact binomial 95\% confidence intervals were calculated for $p_{0}$ and approximate $95 \%$ confidence intervals based on asymptotic standard errors were calculated for the percentage positive agreement, the percentage negative agreement, and kappa (Fleiss et al., 1969; Graham and Bull, 1998).

Cochran's Q, Spearman's rho, and kappa were calculated using commercially available statistical software (version 12.0, SPSS Inc., Chicago, IL), and $P$ values $\leq$ 0.05 were considered statistically significant.

\section{RESULTS}

The results of environmental cultures and serum ELISA testing performed on 23 California dairies are shown in Table 2, along with information on previous MAP infection status based on individual and pooled fecal cultures from 60 animals. Mycobacterium avium ssp. paratuberculosis was cultured from at least 1 environmental sample on 17 (74\%) of 23 farms, compared with the 16 (70\%) of 23 operations that had been identified as infected during previous fecal culture testing of individual animals. With respect to the concurrently performed ELISA testing, there were $\geq 2$ seropositive animals ( $>3 \%$ of the total number tested) on $15(65 \%)$ operations. The proportions of herds that were identified as being infected did not differ among the different testing methods (environmental cultures, previous fecal cultures, and ELISA testing using $>3 \%$ seropositive animals as the cutoff; Cochran's $\mathrm{Q}=0.50,2 \mathrm{df}, P=$ 0.779 ).

Measures of agreement between environmental testing results and the other methods of testing are shown in Table 3. The proportion of seropositive animals on each farm was correlated with the proportion of positive environmental cultures (Spearman's rho $=0.53, P=$ 0.009).

An environmental sample was not collected from the sick/fresh cow pen on 1 of the 23 dairies, but on the remaining 22 operations, environmental samples were collected from all 3 sampling locations (the return alleyway, the alleyways of the sick/fresh cow pen, and the wastewater lagoon). In these 22 herds, the probability of obtaining a positive result was higher for lagoon samples than it was for the 2 alleyway locations, with 14 of $22(64 \%)$ herds having positive lagoon samples compared with 8 of $22(36 \%)$ samples with positive results for each of the 2 alleyway sampling locations (Cochran's $\mathrm{Q}=6.55,2 \mathrm{df}, P=0.038$ ). 
Table 2. Results of serologic testing and environmental cultures for Mycobacterium avium ssp. paratuberculosis (MAP) performed on 23 California dairies during 2004, with information on prior infection status based on individual and pooled fecal cultures performed during 2001

\begin{tabular}{|c|c|c|c|c|c|c|c|}
\hline \multirow[b]{2}{*}{ Herd ID } & \multicolumn{2}{|c|}{ ELISA } & \multicolumn{4}{|c|}{ Environmental cultures $^{2}$} & \multirow{2}{*}{$\begin{array}{l}\text { Prior MAP } \\
\text { infection } \\
\text { status }^{3}\end{array}$} \\
\hline & $\begin{array}{l}\text { No. positive/ } \\
\text { No. tested }\end{array}$ & $\begin{array}{l}\% \\
\text { Positive }^{1}\end{array}$ & FA & RA & LG & $\begin{array}{l}\text { No. positive/ } \\
\text { No. tested }\end{array}$ & \\
\hline 201 & $0 / 57$ & 0.0 & - & - & - & $0 / 3$ & - \\
\hline 203 & $0 / 60$ & 0.0 & - & - & - & $0 / 3$ & + \\
\hline 209 & $0 / 60$ & 0.0 & - & - & + & $1 / 3$ & + \\
\hline 211 & $0 / 60$ & 0.0 & - & - & + & $1 / 3$ & + \\
\hline 213 & $1 / 65$ & 1.5 & + & + & - & $2 / 3$ & + \\
\hline 217 & $1 / 61$ & 1.6 & $\mathrm{NC}^{4}$ & + & + & $2 / 2$ & + \\
\hline 221 & $1 / 60$ & 1.7 & - & - & - & $0 / 3$ & - \\
\hline 216 & $1 / 60$ & 1.7 & - & - & + & $1 / 3$ & + \\
\hline 207 & $2 / 60$ & 3.3 & - & - & - & $0 / 3$ & - \\
\hline 210 & $2 / 60$ & 3.3 & - & - & - & $0 / 3$ & + \\
\hline 214 & $2 / 60$ & 3.3 & - & - & + & $1 / 3$ & - \\
\hline 226 & $2 / 60$ & 3.3 & + & - & + & $2 / 3$ & + \\
\hline 222 & $2 / 59$ & 3.4 & - & - & + & $1 / 3$ & + \\
\hline 227 & $3 / 60$ & 5.0 & - & - & + & $1 / 3$ & + \\
\hline 230 & $9 / 164$ & 5.5 & - & + & + & $2 / 3$ & + \\
\hline 218 & $4 / 60$ & 6.7 & - & - & - & $0 / 3$ & - \\
\hline 204 & $4 / 60$ & 6.7 & + & - & - & $1 / 3$ & - \\
\hline 215 & $4 / 60$ & 6.7 & - & + & + & $2 / 3$ & - \\
\hline 225 & $4 / 60$ & 6.7 & + & + & + & $3 / 3$ & + \\
\hline 228 & $4 / 60$ & 6.7 & + & + & + & $3 / 3$ & + \\
\hline 212 & $11 / 134$ & 8.2 & + & + & + & $3 / 3$ & + \\
\hline 219 & $5 / 60$ & 8.3 & + & + & + & $3 / 3$ & + \\
\hline 208 & $6 / 60$ & 10.0 & + & + & + & $3 / 3$ & + \\
\hline
\end{tabular}

${ }^{1}$ Using an ELISA sample-to-positive (S/P) ratio of $\geq 0.25$ as the cutoff value.

${ }^{2} \mathrm{FA}=$ Fresh alleyway (alleyway sample from the sick/fresh cow pen); RA = return alleyway (sample from the return lane exiting the milking parlor); $L G=$ lagoon (water sample from the wastewater lagoon).

${ }^{3}$ Based on $\geq 1$ positive result from individual and pooled fecal cultures of 60 cows.

${ }^{4} \mathrm{NC}=$ Not collected.

\section{DISCUSSION}

Culture of environmental samples collected from 3 locations on each farm identified a similar proportion of herds as being infected with MAP as did previous fecal culture testing and concurrent ELISA testing of $\geq 60$ animals in each herd. Of the testing methods that were used, environmental sampling identified a higher proportion of herds as being infected $(74 \%)$ than either previous fecal culture testing of individual cattle $(70 \%)$ or concurrent ELISA testing with $>3 \%$ seropositive ani- mals used as the cutoff (65\%). The ELISA testing using $\geq 1$ seropositive animal as the cutoff would have identified a higher proportion of herds as being infected (83\%), but the $3 \%$ cutoff was preferred because ELISA has lower specificity than fecal culture. In a recent study, the specificity of the Idexx ELISA varied from 84 to 100\% across 7 Johne's disease program level-4 herds in Minnesota, with an overall estimate of $94.9 \%$ (Collins et al., 2005).

Measures of agreement with environmental sampling were higher for the results of fecal culture testing

Table 3. Measures of agreement (95\% confidence interval) between environmental sampling and other testing methods for the classification of herd-level Mycobacterium avium ssp. paratuberculosis infection status on 23 California dairies

\begin{tabular}{lcccc}
\hline & \multicolumn{4}{c}{ Measure of agreement ${ }^{1}$} \\
\cline { 2 - 5 } Testing method & $\mathrm{p}_{\mathrm{o}}$ & $\mathrm{p}_{\mathrm{pos}}$ & $\mathrm{p}_{\text {neg }}$ & $\kappa$ \\
\hline ELISA testing of $\geq 60$ animals & 65 & 75 & 43 & 0.19 \\
$\quad$ cutoff $>3 \%$ seropositive results) & $(43,84)$ & $(58,92)$ & $(10,75)$ & $(-0.23,0.60)$ \\
Previous fecal culture of 60 animals & 70 & 85 & 62 & 0.47 \\
$\quad$ cutoff $\geq 1$ positive culture) & $(47,87)$ & $(72,98)$ & $(30,93)$ & $(0.07,0.86)$ \\
\hline
\end{tabular}

${ }^{1} p_{o}=$ Percentage observed agreement; $p_{p o s}=$ percentage positive agreement; $p_{\text {neg }}=$ percentage negative agreement; and $\kappa=$ kappa statistic. 
that had been performed on 60 animals in each herd 2 to $3 \mathrm{yr}$ previously than for the results of concurrent ELISA testing. It is not surprising that environmental sampling and previous fecal culture testing would provide similar results, despite the lag in testing periods, because both are culture-based methods and simulation studies suggest that even when preventive management practices are implemented to control paratuberculosis, the within-herd prevalence decreases slowly over several years (Groenendaal et al., 2002).

Using the scale recommended by Landis and Koch (1977), the kappa value measuring the agreement of environmental cultures with serology using $>3 \%$ seropositive animals as a cutoff for classifying herds as infected would be considered "slight," whereas the kappa comparing environmental cultures with previous fecal culture testing would be considered "moderate." The kappa statistic is known to be sensitive to both prevalence and marginal heterogeneity in $2 \times 2$ tables, with values of kappa tending toward zero when the prevalence is close to either zero or one, and tending toward one when the apparent prevalence differs between methods (Thompson and Walter, 1988; Feinstein and Cicchetti, 1990). In the current study, the proportion of positive results did not differ significantly between testing methods, but the percentage of infected herds was relatively high (with only one herd having a negative result on all tests), which may have decreased the observed kappa values. The estimates of percentage positive agreement were higher than those for percentage negative agreement, which was also a reflection of the relatively high proportion of MAP-infected herds.

Even with a sample size of only 3 environmental cultures per operation, there was a significant positive correlation between the proportion of positive environmental cultures and the proportion of seropositive animals. This suggests that in addition to providing information about whether MAP is present or absent on a farm, environmental cultures may also provide a qualitative estimate of within-herd prevalence. Raizman et al. (2004) found that there was an association between the maximum number of colonies per tube cultured from environmental samples and the prevalence of positive fecal pools. Because colony counts were not available for environmental cultures in the present study, we were unable to evaluate whether this measure was similarly related to seroprevalence.

Among the 3 environmental sampling locations, lagoon water was significantly more likely to yield a positive result than were samples collected from the sick/ fresh cow pen or from the alleyway exiting the milking parlor. In environmental sampling conducted as part of the National Animal Health Monitoring System's
Dairy 2002 study, 69 of $98(70.4 \%)$ herds that were tested had MAP identified in at least one environmental sample, with $47.4 \%$ of lagoon samples testing positive (USDA, 2005a). Wastewater lagoons were also identified as one of the most common areas to be contaminated with MAP in Minnesota dairies (Raizman et al., 2004). Lagoons offer convenient access to composite manure sample from the entire herd, and may also serve as an important link in the chain of MAP transmission on some operations. Using recycled lagoon water to flush barn alleyways is a common practice on large western US dairies. Of the operations that were enrolled in the current study, heifers younger than 6 mo of age were exposed to recycled lagoon water used for flushing on 11 of the $23(48 \%)$ farms. Although it is unknown how long MAP may survive in wastewater lagoons, previous studies have demonstrated the ability of MAP to survive in pond and tap water for 9 and 17 mo, respectively (Lovell et al., 1944; Larsen et al., 1956).

The cost of environmental sampling with collection of 3 samples per farm was low relative to other methods of determining herd infection status. In the California Animal Health and Food Safety Laboratory System, charges for performing Johne's ELISA testing and fecal cultures are currently $\$ 5$ and $\$ 16$, respectively, for instate submissions; and the cost of sample collection and preparation was estimated at $\$ 2$ for individual serum and fecal samples, and $\$ 10$ for pooled and environmental samples. The overall cost of collecting and culturing 3 environmental samples per farm was approximately $[3 \times \$ 16 /$ culture $]+[3 \times \$ 10 /$ sample (handling) $]=\$ 78$, compared with $[60 \times \$ 5 /$ ELISA $]+[60 \times \$ 2 /$ sample (handling)] $=\$ 420$ for ELISA testing of 60 animals. The overall cost of submitting individual fecal samples from 60 animals was approximately [60 $\times \$ 16 /$ culture $]+[60$ $\times \$ 2 /$ sample (handling) $]=\$ 1,080$. The additional pooled fecal samples (10 cows/pool) from 60 cows cost approximately $[6 \times \$ 16 /$ culture $]+[6 \times \$ 10 /$ sample (handling) $]=$ $\$ 156$. Even if the number of environmental samples was doubled to enhance the sensitivity of detection, the overall cost of environmental sampling would be lower than for ELISA or fecal culture testing of 60 individual animals, and equivalent to the cost of using only pooled fecal culture testing of 60 cows (10 cows/pool).

One concern with using environmental sampling for the determination of herd infection status is the possibility that MAP may be detected in the environment without being present in the cattle herd. Although MAP is classified as an obligate pathogen and is not believed capable of replicating outside a suitable host, infection has been identified in a number of wild ruminant species and in rabbits captured near infected farms (Manning and Collins, 2001). Nonetheless, the likelihood that sylvatic hosts would contribute substantially to 
manure samples collected from cow alleyways and wastewater lagoons is minimal. More practical questions may be related to the length of time that MAP can survive in the wastewater lagoon after infection has been eliminated from animals in a herd, and whether MAP is capable of replicating in water-borne amoebas as has been reported for other $M$. avium strains (Cirillo et al., 1997). Regardless, the presence of MAP in the lagoon could be considered an indicator of the potential for ongoing MAP transmission, particularly in those herds that use recycled lagoon water for flushing alleyways and barns.

The large free-stall dairies that were evaluated in the present study may not be typical of herds found in many areas of the United States, and central California's Mediterranean-style climate may limit the generalization of these findings. Most of the enrolled herds were infected with MAP, although they represented a range of within-herd seroprevalences similar to those that have been reported for infected herds both in California (Adaska and Anderson, 2003) and other areas of the country (Johnson-Ifearulundu and Kaneene, 1999; Hirst et al., 2004). Including additional uninfected herds would have improved the precision of the estimated agreement measures, but there are currently no dairies enrolled in the herd classification component of California's Voluntary Johne's Disease Control Program from which verified low-risk or paratuberculosisfree herds could be selected.

\section{CONCLUSIONS}

Culture of environmental manure samples collected from 3 locations on each operation provided information about MAP infection status that was comparable to but less costly than concurrent ELISA testing of $\geq 60$ cows and to previous individual and pooled fecal cultures of 60 animals in each herd. Of the sampling locations that were evaluated, lagoon water was significantly more likely to yield a positive result than were samples collected from the sick/fresh cow pen or the alleyway exiting from the milking parlor. The proportion of positive environmental cultures on each farm was significantly associated with the proportion of seropositive cattle, suggesting that in addition to providing information about herd infection status, environmental sampling may also provide a qualitative estimate of withinherd prevalence.

\section{ACKNOWLEDGMENTS}

Supported in part by an Austin Eugene Lyons Fellowship and the Center for Food Animal Health, University of California, Davis.

\section{REFERENCES}

Adaska, J. M., and R. J. Anderson. 2003. Seroprevalence of Johne's disease infection in dairy cattle in California, USA. Prev. Vet. Med. 60:255-261.

Bulaga, L. 1998. US Voluntary Johne's Disease Herd Status Program for cattle. Pages 420-433 in Proc. Annu. Mtg. U.S. Anim. Health Assoc., Minneapolis, MN.

Cicchetti, D. V., and A. R. Feinstein. 1990. High agreement but low kappa: II. Resolving the paradoxes. J. Clin. Epidemiol. 43:551558.

Cirillo, J. D., S. Falkow, L. S. Tompkins, and L. E. Bermudez. 1997. Interaction of Mycobacterium avium with environmental amoebae enhances virulence. Infect. Immun. 65:3759-3767.

Cohen, J. 1960. A coefficient of agreement for nominal scales. Educ. Psychol. Meas. 20:37-46.

Collins, M. T., S. J. Wells, K. R. Petrini, J. E. Collins, R. D. Schultz, and R. H. Whitlock. 2005. Evaluation of five antibody detection tests for diagnosis of bovine paratuberculosis. Clin. Diagn. Lab. Immunol. 12:685-692.

Feinstein, A. R., and D. V. Cicchetti. 1990. High agreement but low kappa: I. The problems of two paradoxes. J. Clin. Epidemiol. 43:543-549.

Fleiss, J. L., J. Cohen, and B. S. Everitt. 1969. Large sample standard errors of kappa and weighted kappa. Psychol. Bull. 72:323-327.

Fleiss, J. L., B. Levin, and M. C. Paik. 2003. The comparison of $M$ matched samples. Pages 389-394 in Statistical methods for rates and proportions. 3rd ed. Wiley, New York, NY.

Fyock, B. A., J. Smith, E. Hoving, Y. Schukken, and R. H. Whitlock. 2005. Whole herd fecal pools to assess herd MAP bio-burden compared to composite environmental samples. Page 44 in Proc. 8th Int. Coll. Paratuberculosis, Copenhagen, Denmark. http:// www.paratuberculosis.org/proc8/8ICP_Abstracts.pdf Accessed Jan. 6, 2006.

Graham, P., and B. Bull. 1998. Approximate standard errors and confidence intervals for indices of positive and negative agreement. J. Clin. Epidemiol. 51:763-771.

Groenendaal, H., M. Nielen, A. W. Jalvingh, S. H. Horst, D. T. Galligan, and J. W. Hesselink. 2002. A simulation of Johne's disease control. Prev. Vet. Med. 54:225-245.

Hendrick, S. H., T. E. Duffield, D. E. Kelton, K. E. Leslie, K. D. Lissemore, and M. Archamboult. 2005. Evaluation of enzymelinked immunosorbent assays performed on milk and serum samples for detection of paratuberculosis in lactating dairy cows. J. Am. Vet. Med. Assoc. 226:424-428.

Hirst, H. L., F. B. Garry, P. S. Morley, M. D. Salman, R. P. Dinsmore, B. A. Wagner, K. D. McSweeney, and G. M. Goodell. 2004. Seroprevalence of Mycobacterium avium subsp. paratuberculosis infection among dairy cows in Colorado and herd-level risk factors for seropositivity. J. Am. Vet. Med. Assoc. 225:97-101.

Johnson-Ifearulundu, Y., and J. B. Kaneene. 1999. Distribution and environmental risk factors for paratuberculosis in dairy cattle herds in Michigan. Am. J. Vet. Res. 60:589-596.

Landis, J. R., and G. G. Koch. 1977. The measurement of observer agreement for categorical data. Biometrics 33:159-174.

Larsen, A. B., R. S. Merkal, and T. H. Vardaman. 1956. Survival time of Mycobacterium paratuberculosis. Am. J. Vet. Res. 17:549-551.

Lovell, R., M. Levi, and J. Francis. 1944. Studies on the survival of Johne's bacilli. J. Comp. Pathol. 54:120-129.

Manning, E. J., and M. T. Collins. 2001. Mycobacterium avium subsp. paratuberculosis: Pathogen, pathogenesis and diagnosis. Rev.Off. Int. Epizoot. 20:133-150.

Raizman, E. A., S. J. Wells, S. M. Godden, R. F. Bey, M. J. Oakes, D. C. Bentley, and K. E. Olsen. 2004. The distribution of Mycobacterium avium ssp. paratuberculosis in the environment surrounding Minnesota dairy farms. J. Dairy Sci. 87:2959-2966.

Stabel, J. R. 1997. An improved method for cultivation of Mycobacterium paratuberculosis from bovine fecal samples and comparison to three other methods. J. Vet. Diagn. Invest. 9:375-380.

Tavornpanich, S., I. A. Gardner, R. J. Anderson, S. Shin, R. H. Whitlock, T. Fyock, J. M. Adaska, R. L. Walker, and S. K. Hietala. 2004. Evaluation of microbial culture of pooled fecal samples 
for detection of Mycobacterium avium subsp. paratuberculosis in large dairy herds. Am. J. Vet. Res. 65:1061-1070.

Thompson, W. D., and S. D. Walter. 1988. A reappraisal of the kappa coefficient. J. Clin. Epidemiol. 41:949-958.

USDA. 2005b. Uniform program standards for the voluntary bovine Johne's disease control program. APHIS 91-45-016. USDAAPHIS-VS, Washington, DC.

USDA. 2005a. Pages 126-130 in Johne's disease on U.S. dairy operations, 2002. \#N427.0205. USDA-APHIS-VS, CEAH, NAHMS, Fort Collins, CO.

van Schaik, G., S. M. Stehman, Y. H. Schukken, C. R. Rossiter, and S. J. Shin. 2003. Pooled fecal culture sampling for Mycobacterium avium subsp. paratuberculosis at different herd sizes and prevalence. J. Vet. Diagn. Invest. 15:233-241.

Vary, P. H., P. R. Andersen, E. Green, J. Hermon-Taylor, and J. J. McFadden. 1990. Use of highly specific DNA probes and the polymerase chain reaction to detect Mycobacterium paratuberculosis in Johne's disease. J. Clin. Microbiol. 28:933-937.

Wells, S. J., R. H. Whitlock, B. A. Wagner, J. Collins, F. Garry, H. Hirst, J. Lawrence, W. J. Saville, and A. L. Naugle. 2002. Sensitivity of test strategies used in the Voluntary Johne's Disease Herd Status Program for detection of Mycobacterium paratuberculosis infection in dairy cattle herds. J. Am. Vet. Med. Assoc. 220:1053-1057. 